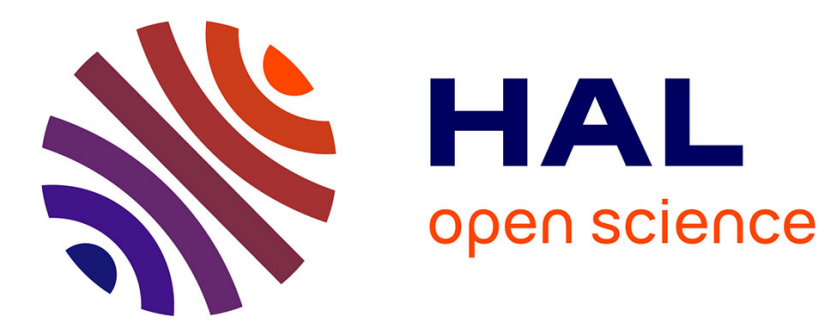

\title{
Multi-Agent Activity-Based Simulation of a Future Neighborhood
}

Younes Delhoum, Rachid Belaroussi, Francis Dupin, Mahdi Zargayouna

\section{To cite this version:}

Younes Delhoum, Rachid Belaroussi, Francis Dupin, Mahdi Zargayouna. Multi-Agent Activity-Based Simulation of a Future Neighborhood. KES-AMSTA 2021, 15th KES International Conference Agents and Multi-Agent Systems: Technologies and Applications, Jun 2021, Virtuel, France. pp. 501-510. hal-03363823

\section{HAL Id: hal-03363823 https://hal.science/hal-03363823}

Submitted on 4 Oct 2021

HAL is a multi-disciplinary open access archive for the deposit and dissemination of scientific research documents, whether they are published or not. The documents may come from teaching and research institutions in France or abroad, or from public or private research centers.
L'archive ouverte pluridisciplinaire HAL, est destinée au dépôt et à la diffusion de documents scientifiques de niveau recherche, publiés ou non, émanant des établissements d'enseignement et de recherche français ou étrangers, des laboratoires publics ou privés. 


\title{
Multi-Agent Activity-Based Simulation of a Future Neighborhood
}

\author{
Younes Delhoum ${ }^{1}$, Rachid Belaroussi ${ }^{1}$, Francis Dupin ${ }^{1}$, Mahdi Zargayouna $^{1}$ \\ ${ }^{1}$ COSYS-GRETTIA, Univ Gustave Eiffel, IFSTTAR, \\ F-77454 Marne-la-Vallée, France \\ \{younes.delhoum, rachid.belaroussi,francis.dupin,mahdi.zargayouna\}@univ-eiffel.fr
}

\begin{abstract}
Mobility simulations are an effective tool to forecast the effects of transportation policies. They are a useful part of decision support systems for policy makers. Big real-estate projects, aiming at creating whole new neighborhoods for instance, need this kind of applications to estimate their impacts on the surrounding environment. The potential visits of the various equipments and the public spaces of the new neighborhood can also be estimated with this kind of tools. In this context, agent-based simulation is a relevant model for the design and implementation of transportation applications, that represents travelers individually, together with their perception model of the environment and their internal decision processes. In this paper, we propose a multi-agent activity-based simulation to represent current and future mobility patterns in a city, with or without a new neighborhood. We describe the inputs of the simulation, in terms of networks and travel demand, the behaviors of the agents and the outputs on the laVallée project (France). The first series of experiments demonstrate the potential of the simulation and its benefits for the project managers and the decision-makers in the concerned territories.
\end{abstract}

\section{Introduction}

Large-scale and complex mobility systems could be represented by simulating the behaviors and interactions of self-interested "agents", evolving in a transportation environment, creating a multi-agent system. In [1], the authors state multiple reasons to use multiagent systems in transportation applications. The authors indicate that the solving of several transportation problems with multiagent systems is natural and intuitive. The simulation of passengers mobility is particularly suitable for an agent-based design. Indeed, the objective in these simulations is to take into account human behaviors, interacting in an open, dynamic and complex environment [2]. This configuration obey to the general definitions of agents as entities that are situated in some environment, that are capable of autonomous action on it [3], that can perceive this environments and have a partial and incomplete perception of it [4].

Among multi-agent models, there is a class called activity-based models, that address specifically the need for realistic representation of travel demand and 
the human behavior in a mobility context. The activity-based modeling (ABM) framework was originally developed in response to demands for more realistic travel demand models which are capable of analyzing a wider range of transportation policies. These models are capable of evaluating travel demand and transportation supply management strategies, such as road pricing, and behavior modification programs (flexible scheduling, ride-sharing), in a more efficient way than the previous generation of aggregate flow models, which generally focus on evaluating network capacity improvement.

In activity-based models, unlike traditional analytical trip-based models, the travel demand originates from the needs of the traveler to pursue some activities that are distributed in time and space. The understanding of the travel decisions becomes hence secondary compared to the fundamental understanding of the agents activities [5]. Based on this methodological choice, activity-based models have been proposed, such as TRANSIMS [6] and ALBATROSS [7].

The LaVallée project (Paris Area, France) is a large construction project, to be delivered in 2024, with an area of about $500 \mathrm{~m} \times 400 \mathrm{~m}$, and is part of a larger city called Chatenay-Malabry. It will house 6000 residents $(20 \%$ of the city population), a large set of services ranging from a shopping street, a mall, schools and office spaces for 2000 employees; and the district is designed to be fully integrated in the rest of the city. The effects of this new neighborhood on the surrounding city is complex to forecast. One of the reasons is that the new district might imply changes in the behaviors of the city users, that are difficult to model with traditional modeling paradigms. This is the reason why we have chosen to model the city, with and without the new neighborhood, with a multi-agent activity-based simulation.

The remainder of this paper is structured as follows. Section 2 is the related work section. Section 3 describes the model of the simulation. Section 4 provides the first results of our simulations. Finally, section 5 concludes the paper.

\section{Related Work}

The modeling of mobility has been long dominated by the modeling approach called the four step model. In this approach, trip origin-destination (O-D) is the principle database. However, the valid representation of underlying travel behavior in this conventional trip-based model of travel demand forecasting has never really been proved [8]. In contrast, activity-based models use a sequence of activities to represent the mobility behavior of the population. Transportation demand is the result of the agents need to satisfy their goals via activities taking place in different locations and times. The activity-based approach considers contextualized individual trips, making it possible to represent more realistic travel behaviors.

When representing mobility behaviors, first generations of simulations were governed by a centralized top-down process. Proactive interactions and communication between individuals were not possible. Agent-based modeling and simulation came as an answer to this limitation. Multi-agent systems are useful 
when studying complex and self-organizing systems [9]. The models in this category are based on the concept of autonomous entities, called agents, that are situated in a shared observable environment, that they perceive and where they can act upon, in order to achieve their own goals. Several agent-based models exist in the literature, such as AgentPolis [10], SUMO [11], Aimsun [12], SM4T [13], etc.

In this paper, we use a specific kind of multi-agent models, called activitybased models [14]. In this category, the agents plan and execute activity schedules. Each activity is of a specific type (e.g., work, school or shop) and has associated start times and durations. Trips between each pair of activities are specified by their transport mode (e.g., car or public transport). Models like SCHEDULER [15], TRANSIMS [6], CEMDAP [16], SacSim [17], actiTopp [18], the two-level dynamic model [19] or the walk-bicycle ABM [20] are examples of existing activity-based models.

\section{The Model}

\subsection{Overview}

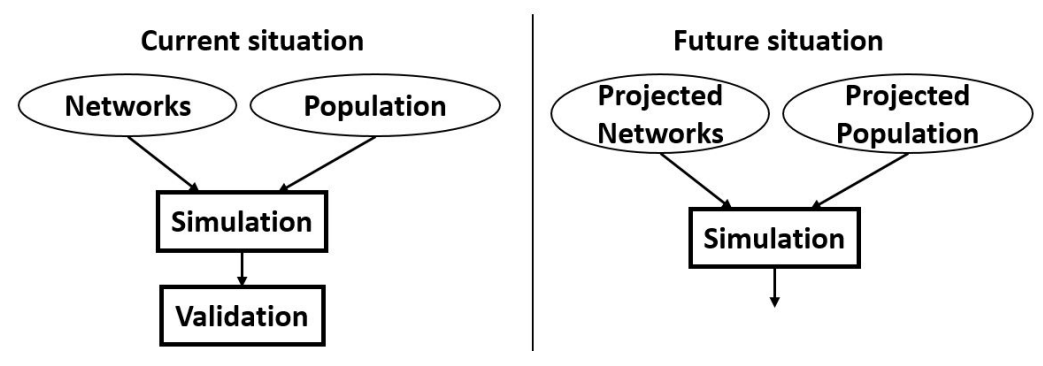

Fig. 1. Overview of the methodology

The main idea behind our process to forecast the future impact of the new neighborhood (LaVallée) on the surrounding city is (cf. Fig. 1):

1. to perform a valid simulation of the current situation (without LaVallée)

2. to perform a simulation of the hypothetic future situation, by modifying the inputs

Each one of these two simulations is performed following the process described in Fig. 2. The inputs of the simulation are:

1. The population of the area is synthesized, to represent the actual users of the network, notably including their activities

2. Schedules are generated for the population

3. Several runs are executed to reschedule the activities of the agents until reaching convergence

These steps are described in more details in the following paragraphs. 


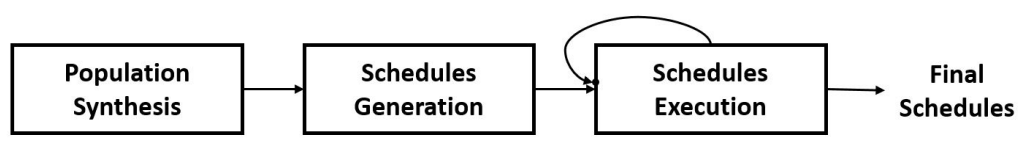

Fig. 2. Overview of a simulation workflow

\subsection{Input data}

The input of the current situation simulation are:

1. The global survey data for transport from household census.

2. The road network: by default OpenStreetMap (OSM) files.

3. Public Transportation (PT) network: the PT (bus/train/tram) stops location and times from General Transit Feed Specification (GTFS), the PT routes were generated following the stops-sequence using a shortest path algorithm, compared and corrected manually.

The input of the future situation simulation are:

1. The current situation data, plus the population data based on a survey of the real-estate developer (see Figure 3).

2. The road network: The current situation data, plus the road network and walk network of the new neighborhood.

3. The current situation data, plus a future tramway line (T10, expected in 2023).

4. The locations of a set of activities: school, work, leisure and shopping.

The detailed process of population synthesis, together with all the used data can be found in [21].

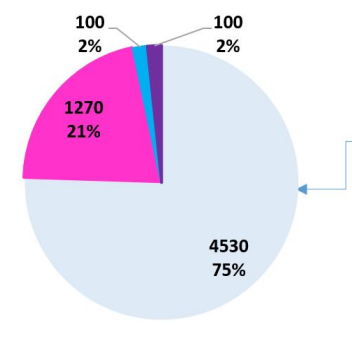

Others Education Work Windergarten

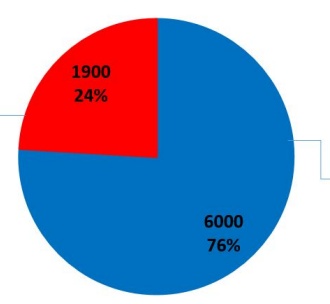

- Residents Externals

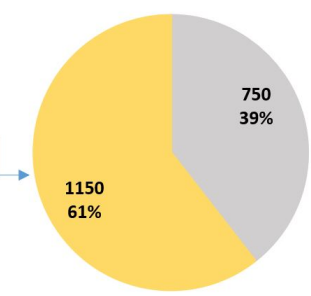

$\approx$ Car $₫$ Walk \& PT

Fig. 3. LaVallée population data. (Left): the residents trip-purposes. (Center): the proportion of users from LaVallée distributed between residents and externals. (Right): the external workers transport mode-share5 


\subsection{Agents behaviors}

The traveler agent iteratively tries to improve a score associated to its current plan, until no improvement is observed. Indeed, each traveler agent has the objective of performing its sequence of activities. It tries to implement a current plan and gets a corresponding score. The score depends on the travel time of the agent and the duration of its activities. To do so, each agent has an internal scoring function, which stores the activities start and end times, together with legs starts and ends.

The implementation of each agent currently scheduled plan is dependent on the links dynamics. The physics of a link is determined by the free speed link travel time, its capacity and storage capacity. These quantities are computed from the link length, the number of lanes, and the link's maximum speed. The capacity is the classic maximum number of vehicles allowed to leave the link per time step, and the storage capacity of a link is the maximum number of vehicles that can be placed on the link.

\section{Experiments}

\subsection{Case Study}

Our objective is to assess the impact of the LaVallée project on the whole city. We take into account the flow of external visitors, estimated realistically based on the pre-project movements in the areas of influence of LaVallée. We define the catchment area of LaVallée district as the area that can be reached by future potential visitors. Potential visits correspond to a need for a specific purpose (shopping, leisure, school) that are currently done outside of the district, but would be more convenient if realized in LaVallée, when the real estate will be in place. Figure 4 indicates the location of the district of interest, and the catchment area where LaVallée would attract a population that will use its services. This area is defined as the zone accessible in less than $15 \mathrm{~min}$, by private cars or public transportation. This definition imply a rather large area.

\subsection{Setup}

The population of the catchment area, composed of 126,151 agents, is simulated. It represents around $8 \%$ of the department (Hauts-de-Seine) population.

The simulation has a set of parameters, such as the replanning (rescheduling) strategies probabilities, the focus is on two strategies: ReRoute and TimeAllocationMutator. By selecting the first (second) strategy, the agent has to change its travel route (start and end time of its activities) for the current iteration. A third strategy is Best Score, the agent plan will remain the same. Each strategy has a selection probability $P$, the simulation runs using the following probabilities: $P_{\text {ReRoute }}(20 \%), P_{\text {TimeAllocationMutator }}(10 \%), P_{\text {BestScore }}(70 \%)$.

All simulations were performed with the MATSim platform [22], the details about the redefining of the workflow on MATSim is in Figure 5. 

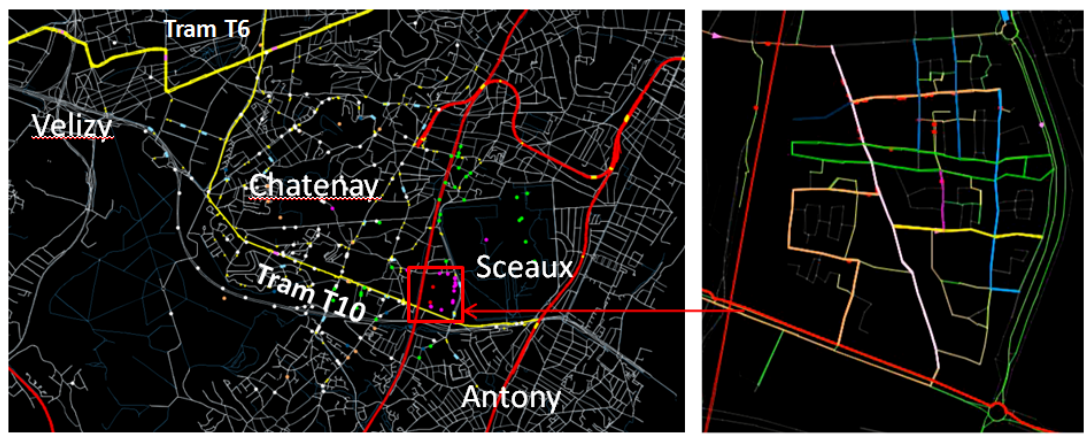

Fig. 4. (Left): the catchment area. (Right): LaVallée future road network (walk, bicycle and cars).

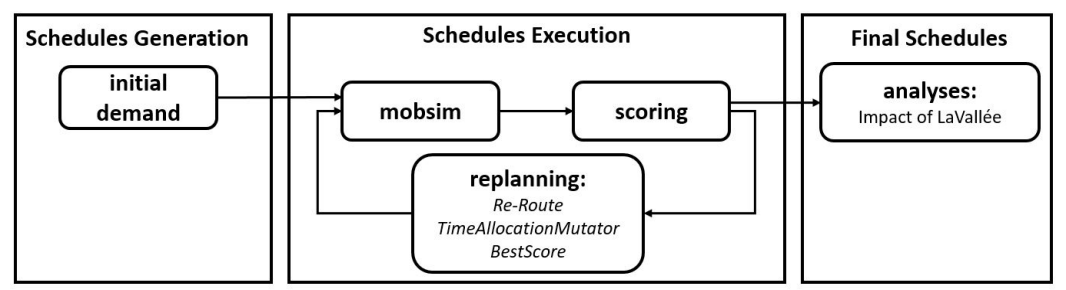

Fig. 5. Redefining our workflow on MATSim platform. (Left): the generated schedules are represented by the initial demand. (Center): the schedules executions are represented by the three steps: mobility simulation, scoring and replanning with its strategies. (Right): the final schedules are analysed to extract the impact of the new neighborhood on the whole population

\subsection{Results}

Two main scenarios were simulated: the current situation and future situation.

Current situation In the first scenario, we are interest in the current situation, without the presence of LaVallée. In this case, each agent has only one plan (original plan). The type of activity (trip-purpose) and the mode-share distributions are described in Figure $6(\mathrm{a}, \mathrm{b})$.

To validate our simulation, we focus on four activities: work, education, leisure and shopping. The results show very close distributions (for these activities) with reference distributions (Enquête Globale de Transport [23]), the results are in Figure 6 (c).

However, we notice some differences in some activities. The first is the work activity, these differences are mainly related to the large number of workplaces (workers) in other areas of the department (such as: La-Defense). The education activity follows the same pattern, more schools are in the other of Hauts-deSeine (comparing with catchment area). For the remaining activities (leisure and shopping), similar distributions were found. 
Multi-Agent Activity-Based Simulation of a Future Neighborhood

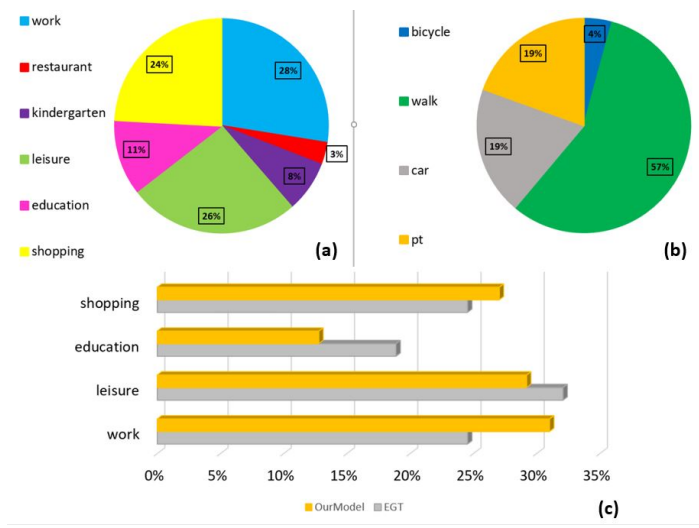

Fig. 6. (a) Trip purpose, (b) transport mode share in the district catchment area and (c) activity distribution (OurModel vs EGT)

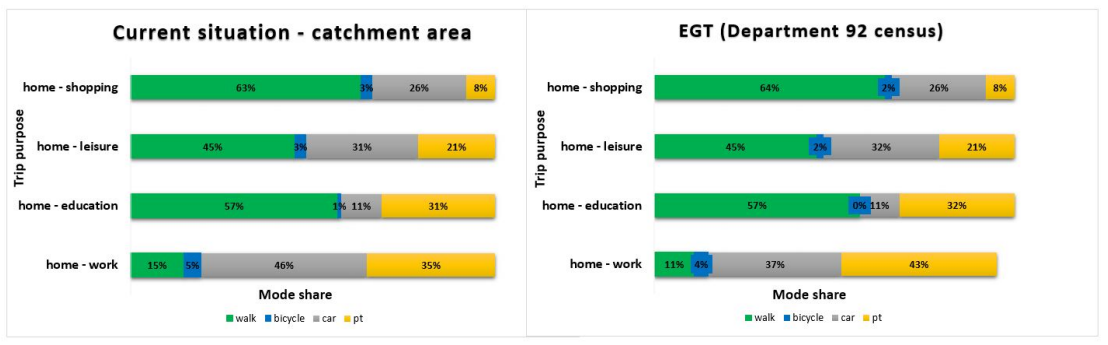

Fig. 7. Transportation mode share for different trip purposes. (Left): the extracted result from the current situation simulation's of the catchment area. (Right): data from EGT of the whole department 92 [22]. Travel mode shares from the model are similar to the 92 census with minor differences.

Figure 7 shows a comparison on values of transport mode share for previous trip purposes. Four transport mode were considered: public transportation (pt), private vehicle (car), walking (walk) and cycling (bicycle).

With these results, we consider that we represent quite accurately the current situation, and can move forward with executing future scenarios.

Future situation By considering the future situation, including the new neighborhood, each traveler can alter its plans, the idea is to propose for each potential visitor (a traveler has leisure or shopping as activity), to do that activity in LaVallée. Statistically, $48 \%(60,084)$ of the agents have leisure as activity, $44 \%$ $(56,009)$ have shopping and $68 \%(85,407)$ have leisure or shopping.

By using the same simulation settings of the current situation simulation, we study the proportion of potential visitors. The simulation show that: $6.61 \%$ $(3,973)$ of the potential visitors come for leisure activity, $6.30 \%(3,531)$ for shopping and $7.92 \%(6,767)$ for leisure or shopping (see Figure 8). 
Younes Delhoum, Rachid Belaroussi, Francis Dupin, Mahdi Zargayouna

\begin{tabular}{|c|c|c|c|}
\hline Trip-purpose & External-visitors & Frequency (activity) & (\%) External-visitors \\
\hline leisure & 3973 & 60084 & $6.61 \%$ \\
\hline shopping & 3531 & 56009 & $6.30 \%$ \\
\hline leisure or shopping & 6767 & 85407 & $7.92 \%$ \\
\hline
\end{tabular}

Fig. 8. Proportion of external visitors who's switch into LaVallée

Another objective of this work is to study the benefits of the new neighborhood. As a measure, we consider the gain that an agent would earn from executing an activity in LaVallée (in term of score). This gain is equal the difference in score between LaVallée selected-plan and main-plan (in the current situation). The results show that the visitors $(6,767)$ have an average gain of 5.63 (score points) with a median gain of 2.41. At the population scale, the global gain is around 0.3 , it can represent the benefit in travel time (a faster route), and time-choice (duration, start and end time) of the activities. The quantification of the gain (in terms of score) depends on the simulation functions.

\begin{tabular}{|c|c|c|c|c|c|c|}
\hline Trip-purpose & Mean & Min & $Q_{1}$ & Median $\left(Q_{2}\right)$ & $Q_{3}$ & Max \\
\hline leisure & 5.63 & 0.00 & 0.89 & 2.43 & 5.30 & 511.72 \\
\hline shopping & 4.94 & 0.00 & 0.86 & 2.28 & 5.14 & 454.18 \\
\hline leisure or shopping & 5.49 & 0.00 & 0.87 & 2.41 & 5.32 & 511.72 \\
\hline
\end{tabular}

Fig. 9. Five-number summary and mean values, for the agent gain

To better analyze the benefit value, the five-number summary is calculated. The results show that almost $25 \%$ of visitors have gains that are higher than the mean value ( 5 points), more details are in Figure 9.

The proportion of visitors depends mainly on the replanning strategies, to understand better the behaviour of agents, we decide to vary the selection probability $P$ of ReRoute (S1) and TimeAllocationMutator (S2) strategies, the remaining probability is set to BestScore strategy.

The results show that $4.01 \%(3,425)$ of agents come to LaVallée (with $P_{S 1}=$ $\left.P_{S 2}=0 \%\right)$, they are whose living close to LaVallée. By increasing the probability of $S 1$, the number of visitors increases very slowly, it passes from $4.01 \%$ for $\left(P_{S 1}\right.$ $=0 \%)$ to $5.05 \%$ for $\left(P_{S 1}=44 \%\right)$. This slow rise is due the localisation of LaVallée in the catchment area (the furthest location is at 15 minutes by car). By changing the route, the traveler can reduce its travel-time but with a little time, which is not enough to boost the score and then selecting the LaVallee-plan.

However, the results show that $S 2$ has more impact on visitors' decision, by changing the probability $P_{S 2}$, the proportion of visitors pass from $4.01 \%(3,425)$ to $15.39 \%(13,142)$ with $P_{S 2}$ variants from $0 \%$ to $46 \%$. The switch in the starttime and duration impact the plan (score), which promotes the neighborhood activities. LaVallée-plans become a better alternative, especially for the agents who live a little further. The potential visit for both cases is presented in Fig- 
Multi-Agent Activity-Based Simulation of a Future Neighborhood

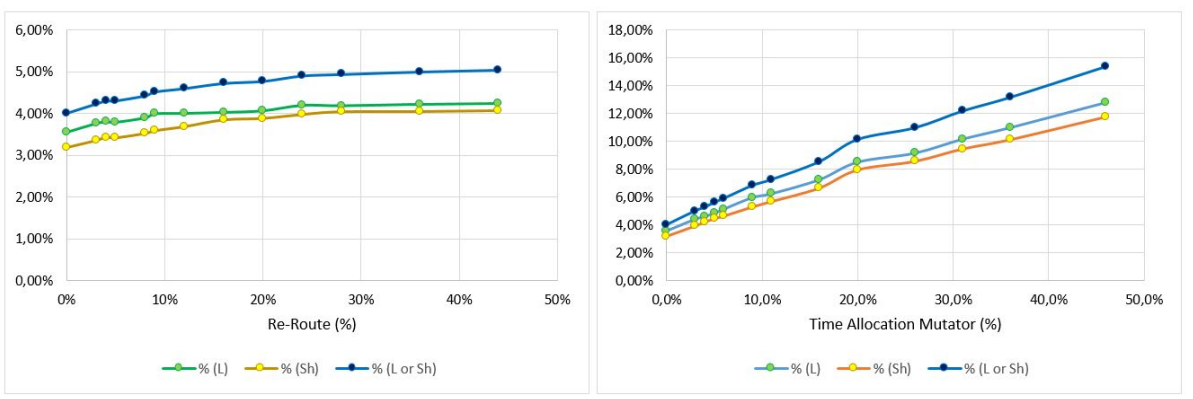

Fig. 10. (Left): Changing the trip-route (ReRoute) has a little impact on the proportion of potential visitors. (Right) the changing in the time (duration) of an activity has a large impact on the proportion of potential visitors, this proportion increase linearly with the selection probability of TimeAllocationMutator

ure 10. Both strategies (in particular S2) could be used to calibrate the model once the counts are realized.

\section{Conclusion}

In this paper, we describe a multi-agent activity-based simulation to forecast the impact of a future neighborhood on the mobility on the scale of a city. The activity-based modeling allows for a fine-grained description of the travelers and a flexible behavior of the system. We first implement and validate a simulation of the current situation, then we implement the simulation representing the future situation. The results show that our model represents the current situation with some minor differences, which is related mainly to the region's infrastructure. Based on the simulations, a potential part of travelers will join LaVallée for an activity, it improves their activity-plan score, the volume of travelers depends mainly on the time and duration of the activities.

\section{References}

1. Bazzan, A.L., Klügl, F.: A review on agent-based technology for traffic and transportation. The Knowledge Engineering Review 29(03) (2014) 375-403

2. Bessghaier, N., Zargayouna, M., Balbo, F.: Management of urban parking: an agent-based approach. In: Artificial Intelligence: Methodology, Systems, and Applications. Springer Berlin Heidelberg (2012) 276-285

3. Wooldridge, M., Jennings, N.R.: Intelligent agents: Theory and practice. The knowledge engineering review 10(02) (1995) 115-152

4. Ferber, J.: Multi-Agent Systems : An introduction to Distributed Artificial Intelligence. Volume 1. Addison-Wesley Longman Publishing Co., Inc. (1999)

5. Jones, P.E., Koppelman, F.S.: Activity analysis: State-of-the-art and future directions. In: Developments in dynamic and activity-based approaches to travel analysis. (1990) 
Younes Delhoum, Rachid Belaroussi, Francis Dupin, Mahdi Zargayouna

6. Smith, L., Beckman, R., Baggerly, K.: Transims: Transportation analysis and simulation system. Technical report, Los Alamos National Lab., NM (United States) (1995)

7. Arentze, T., Timmermans, H.: Albatross: a learning based transportation oriented simulation system. Citeseer (2000)

8. McNally, M.G.: The four-step model. Emerald Group Publishing Limited (2007)

9. Zargayouna, M., Trassy, J.S., Balbo, F.: Property based coordination. In: International Conference on Artificial Intelligence: Methodology, Systems, and Applications, Springer (2006) 3-12

10. Jakob, M., Moler, Z., Komenda, A., Yin, Z., Jiang, A.X., Johnson, M.P., Pechoucek, M., Tambe, M.: Agentpolis: towards a platform for fully agent-based modeling of multi-modal transportation (demonstration). In: AAMAS 2012. (2012) 1501-1502

11. Behrisch, M., Bieker, L., Erdmann, J., Krajzewicz, D.: SUMO - simulation of urban MObility - an overview. In: SIMUL 2011, The Third International Conference on Advances in System Simulation. 55-60

12. Barceló, J., Casas, J.: Dynamic network simulation with aimsun. In: Simulation approaches in transportation analysis. Springer (2005) 57-98

13. Zargayouna, M., Othman, A., Scemama, G., Zeddini, B.: Multiagent simulation of real-time passenger information on transit networks. IEEE Intelligent Transportation Systems Magazine 12(2) (2020) 50-63

14. Ben-Akiva, M.E., Bowman, J.L.: Activity based travel demand model systems. In: Equilibrium and advanced transportation modelling. Springer (1998) 27-46

15. Gärling, T., Kwan, M.p., Golledge, R.G.: Computational-process modelling of household activity scheduling. Transportation Research Part B: Methodological 28(5) (1994) 355-364

16. Ziemke, D., Nagel, K., Bhat, C.: Integrating cemdap and matsim to increase the transferability of transport demand models. Transportation research record 2493(1) (2015) 117-125

17. Bradley, M., Bowman, J.L., Griesenbeck, B.: Sacsim: An applied activity-based model system with fine-level spatial and temporal resolution. Journal of Choice Modelling 3(1) (2010) 5-31

18. Hilgert, T., Heilig, M., Kagerbauer, M., Vortisch, P.: Modeling week activity schedules for travel demand models. Transportation Research Record 2666(1) (2017) 69-77

19. Dianat, L., Habib, K.N., Miller, E.J.: Two-level, dynamic, week-long work episode scheduling model. Transportation Research Record 2664(1) (2017) 59-68

20. Aziz, H.A., Park, B.H., Morton, A., Stewart, R.N., Hilliard, M., Maness, M.: A high resolution agent-based model to support walk-bicycle infrastructure investment decisions: A case study with new york city. Transportation research part C: emerging technologies 86 (2018) 280-299

21. Delhoum, Y., Belaroussi, R., Dupin, F., Zargayouna, M.: Activity-based demand modeling for a future urban district. Sustainability 12(14) (2020) 5821

22. Maciejewski, M., Nagel, K.: Towards multi-agent simulation of the dynamic vehicle routing problem in matsim. In: Proceedings of the 9th International Conference on Parallel Processing and Applied Mathematics - Volume Part II. PPAM'11, Berlin, Heidelberg, Springer-Verlag (2012) 551-560

23. Enquête Globale Transport-EGT 2010-Île de France Mobilités-OMNIL-DRIEA. Available online: http://www.omnil.fr/spip.php?article88 (accessed on 31 January 2021). 